\title{
Similar scaling of contralateral and ipsilateral cortical responses during graded unimanual force generation
}

\author{
G. Derosière ${ }^{\mathrm{a}, \mathrm{b}}$, F. Alexandre ${ }^{\mathrm{a}}$, N. Bourdillon ${ }^{\mathrm{a}, 1}$, K. Mandrick ${ }^{\mathrm{a}, \mathrm{c}}$, T.E. Ward ${ }^{\mathrm{b}, 2}$, S. Perrey ${ }^{\mathrm{a}, *}$ \\ a Movement to Health (M2H), Montpellier-1 University, EuroMov, 700 Avenue du Pic Saint Loup, 34090 Montpellier, France \\ b Biomedical Engineering Research Group (BERG), National University of Ireland Maynooth (NUIM), Maynooth, Co Kildare, Ireland \\ c Bodysens, 442 Rue Georges Besse, Immeuble Innovation 3, 30035 Nîmes, France
}

\section{A R T I C L E I N F O}

\section{Article history:}

Accepted 6 February 2013

Available online 13 February 2013

\section{Keywords:}

NIRS

Cortical activation

Muscle force

Ipsilateral

Handgrip

Unimanual task

\begin{abstract}
A B S T R A C T
Hemibody movements are strongly considered as being under the control of the contralateral hemisphere of the cerebral cortex. However, some neuroimaging studies have found a bilateral activation of either the primary sensori-motor (SM1) areas or the rostral prefrontal cortex (PFC), during unimanual tasks. More than just bilateral, the activation of these areas was found to be symmetrical in some studies. However, the symmetrical response remains strongly controversial notably for handgrip force generations. We therefore aimed to examine the bilateral SM1 and rostral PFC area activations in response to graded submaximal force generation during a unilateral handgrip task. Fifteen healthy subjects performed 6 levels of force (ranging from 5 to $50 \%$ of MVC) during a handgrip task. We concomitantly measured the activation of bilateral SM1 and rostral PFC areas through near-infrared spectroscopy (NIRS) and the electromyographic (EMG) activity of the bilateral flexor digitorum superficialis (FDS) muscles. Symmetrical activation was found over the SM1 areas for all the investigated levels of force. At the highest level of force (i.e., 50\% of MVC), the EMG of the passive FDS increased significantly and the ipsilateral rostral PFC activation was found more intense than the corresponding contralateral rostral PFC activation. We suggest that the visuo-guided control of force levels during a handgrip task requires the cross-talk from ipsi- to contralateral SM1 to cope for the relative complexity of the task, similar to that which occurs during complex sequential finger movement. We also propose alternative explanations for the observed symmetrical SM1 activation including (i) the ipsilateral corticospinal tract and (ii) interhemispheric inhibition (IHI) mechanism. The increase in EMG activity over the passive FDS could be associated with a release of IHI at 50\% of MVC. Finally, our results suggest that the greater ipsilateral (right) rostral PFC activation may reflect the greater demand of attention required to control the motor output at high levels of force.
\end{abstract}

(c) 2013 Elsevier Inc. All rights reserved.

\section{Introduction}

The corticospinal tract (CST) is classically depicted as a crossed pathway and subsequently hemibody movements are often considered as emanating from under the control of the contralateral hemisphere of the cerebral cortex. However, the true nature of the CST is more complex, this structure not being a fully crossed pathway (Kuypers, 1985). Those nervous fibers not crossing constitute the ipsilateral CST. Numerous authors have described the role of the latter in the control of unilateral

\footnotetext{
* Corresponding author at: Movement to Health (M2H), Montpellier-1 University, EuroMov, 700 Avenue du Pic Saint Loup, 34090 Montpellier, France. Fax: + 3341175 9050.

E-mail address: stephane.perrey@univ-montp1.fr (S. Perrey).

1 Present address: Institut des Sciences du Mouvement et de la Médecine Sportive (ISMMS), Université de Genève Rue du Conseil Général, 10-1205 Genève, Switzerland.

2 Present address: Schwartz Center for Computational Neuroscience, Institute for Neural Computation, University of California San Diego, La Jolla, CA 92093-0559, USA.
}

hand tasks (Bawa et al., 2004; Brus-Ramer et al., 2009; Wassermann et al., 1991, 1994; Ziemann et al., 1999) and have implicated ipsilateral cortical activation in unimanual control through neuroimaging methods. For instance, Wriessnegger et al. (2008) reported, using the near-infrared spectroscopy (NIRS) technique, a bilateral activation over the primary sensorimotor (SM1) areas during a unilateral finger tapping task, as revealed by an increase in oxyhemoglobin $\left(\mathrm{O}_{2} \mathrm{Hb}\right)$ and a slight decrease in deoxyhemoglobin (HHb). Similarly, Pfurtscheller et al. (2000) found a bilateral electroencephalography (EEG)-measured event-related desynchronization in the lower mu-rhythm during unimanual movement compared to rest.

Whereas the presence of ipsilateral cortical involvement in unimanual tasks is clearly supported through previous studies the extent or magnitude of this relation has still not been definitively established. On the one hand, numerous studies describe the response of the SM1 areas as asymmetric (Catalan et al., 1998; Ehrsson et al., 2000; Kawashima et al., 1996; Tanji et al., 1988), and favor a higher contralateral activation for a large panel of hand tasks. On the other hand, no significant difference between the ipsilateral and the contralateral 
activations of these areas were found during complex sequential finger movements (Verstynen et al., 2005). The high magnitude of the ipsilateral SM1 area activation was related to the role of this area in "shaping precisely" the muscular command originating from the contralateral hemisphere (Davare et al., 2007; Ehrsson et al., 2000) when fine finger control is required. Indeed, according to the transcranial magnetic stimulation study performed by Davare et al. (2007), during unimanual fine finger movements the ipsilateral M1 strongly contributes to the setting of muscle recruitment timing, either through facilitatory or inhibitory mechanisms. In summary, traditional neuroimaging data show that tasks involving a higher complexity in the sequencing of finger movements elicit stronger and less asymmetric activation pattern in motor areas. Nevertheless, similar results were found with functional magnetic resonance imaging (fMRI) for the production of various submaximal levels of force in power-handgrip tasks (Dai et al., 2001), a task which does not require much precision according to phylogenetic and functional considerations (Ehrsson et al., 2000; Napier, 1956). Indeed, based on the findings from Dai et al. (2001), albeit not highlighted by the authors, one may observe a symmetrical activation of the SM1 areas from 20 to $65 \%$ of maximal voluntary contraction (MVC) during a handgrip task. Further, Shibuya et al. (2008) found a NIRS-measured symmetrical M1 activation during the course of a low-intensity fatiguing handgrip task.

It is possible that this symmetrical activation pattern may not be limited to the SM1 areas only. The rostral prefrontal cortex (PFC) areas for example, are ipsilaterally connected to the motor areas through cortico-cortical pathways (Krieghoff et al., 2011) and are involved in the initiation and the control of voluntary movements (Miller and Cohen, 2001). A bilateral activation of the rostral PFC areas has been observed during fatiguing unilateral handgrip tasks (Liu et al., 2003; Mottola et al., 2006). However, the involvement of processes associated with fatigue in these studies and in Shibuya et al. (2008) may obscure the true extent of such bilateral activation. Finally, while previous studies have reported a symmetrical activation of the SM1 areas during various submaximal levels of force (Dai et al., 2001), the corresponding result for the rostral PFC has not been reported to date.

Therefore, we aim to examine the ipsilateral and contralateral activation responses of SM1 and rostral PFC areas to graded levels of force production during a unilateral handgrip task. We suggest that the symmetrical activation observed is not a property of the complex sequential finger movements only. Rather, in accordance with the results of Dai et al. (2001), we hypothesize that contralateral and ipsilateral rostral PFC and SM1 changes operate in a similar way with graded submaximal force generation during a unilateral handgrip task. The new insight revealed by this study may be relevant for the diagnostic evaluation of neurological hand motor assessments. Such a NIRS method could be used to examine the cortical activation during the assessment and at the same time monitor treatment progress with less strenuous procedures than traditional neuroimaging methods (fMRI and EEG).

\section{Materials and methods}

\section{Participants}

Fifteen healthy volunteers took part in the study (aged 28.0 \pm 7.5 years; height $175.5 \pm 5.9 \mathrm{~cm}$; body weight $69.4 \pm 8.9 \mathrm{~kg}$ ). All subjects were right-handed according to the Edinburgh Questionnaire (Oldfield, 1971). None had any sign of neurological, respiratory, and cardiovascular disease or used medication, which might affect brain and muscle functions. Each subject provided written informed consent prior to participation in the study. All procedures were approved by the local ethics committee (CPP Sud-Méditerranée II, number 2010-11-05) and complied with the Declaration of Helsinki for human experimentation.

\section{Protocol and task procedure}

The experiments were conducted in a quiet and dimly lit room. Each subject performed the entire protocol once. The subjects were asked to sit comfortably at a table. They were facing a computer screen with the left forearm resting upon the surface and held in place with straps to prevent extraneous movements during isometric contractions of the right forearm. The dominant hand was held in neutral position in the sagittal plane. The angle of the elbow was set to $110^{\circ}$ for each subject (with $0^{\circ}$ corresponding to the full extension of the arm). The protocol began with a familiarization and warm-up phase with the handgrip task. After individual set-up, the subjects were requested to perform during $5 \mathrm{~min}$ a few static submaximal contractions of the finger flexors in an intermittent mode. Foremost, the subjects produced three MVCs of a 5-s duration followed by $90 \mathrm{~s}$ of passive recovery. Second, each subject underwent an experimental block-paradigm design containing six conditions of static submaximal force levels repeated three times. The target levels of force were set at $5 \%, 10 \%, 20 \%, 30 \%, 40 \%$ and $50 \%$ of MVC. The subjects matched their force with a target force for $30 \mathrm{~s}$ followed by $60 \mathrm{~s}$ of rest. Applying such intervals in the set-up was determined following pilot trials to both maximize NIRS-evoked responses and minimize the occurrence of neuromuscular fatigue. The block paradigms were pseudo-randomized to avoid order and fatigue effects as well. The pseudo-random order prevented immediate repetition of relatively high force levels between conditions and blocks. Immediately after each block, a MVC was carried out to ensure that no neuromuscular fatigue was induced by the successive muscle contractions. To reduce artifacts, the subjects were asked throughout the experimental protocol to minimize head and body movements as well as to breathe as gently and as regularly as possible.

\section{Measurements}

Force

The levels of force were recorded using a handgrip dynamometer (Captels, Saint-Mathieu de Tréviers, France). The force signals were recorded at 1000 samples per second using a data acquisition system (Biopac MP30, System Inc., Santa Barbara, CA). To maintain the level of force correctly, visual feedback was displayed on the computer screen facing the subjects.

\section{Electromyography}

The surface electromyogram (sEMG) of the flexor digitorum superficialis (FDS) muscle of the hand actively involved in the task (right) and the passive (left) hand were recorded using bipolar $\mathrm{Ag} / \mathrm{AgCl}$ electrodes (Contrôle Graphique Medical, Brie-Comte-Robert, France) with a 9-mm diameter at an inter-electrode distance of $20 \mathrm{~mm}$. The skin was shaved, abraded and washed with emery paper and cleaned with $70^{\circ}$ alcohol in order to obtain low impedance between the two bipolar electrodes $(<3 \mathrm{k} \Omega)$. The reference electrode was positioned on the styloid process of the left ulna. The EMG cables were strapped to the chair to prevent movement artifacts. The EMG signals were amplified $(\times 1000)$, measured at a sample rate of 1000 samples per second and synchronized with the force signals using the Biopac MP30 data acquisition system (Biopac System, Inc., Santa Barbara, CA).

\section{Near infrared spectroscopy}

The NIRS technique has been described elsewhere (Elwell et al., 1994) and has been demonstrated previously as relevant for investigating cortical activity during movement generation (Perrey, 2008). NIRS measurements were performed using a continuous wave (CW) multichannel NIRS system (Oxymon Mk III, Artinis, The Netherlands). The sampling rate was set at $10 \mathrm{~Hz}$. This system allowed measurement of changes in optical density at two different wavelengths in the near-infrared range (nominal wavelengths 760 and $850 \mathrm{~nm}$ ) 
before converting them into changes in concentration of $\left[\mathrm{O}_{2} \mathrm{Hb}\right]$ and [HHb]. A subject-specific differential path length factor (DPF) was used for this conversion based on the age of each subject (Duncan et al., 1996). This allowed measurement of the concentration changes of $\left[\mathrm{O}_{2} \mathrm{Hb}\right]$ and $[\mathrm{HHb}]$ in $\mu \mathrm{M}$ unit (Delpy et al., 1988). The inter-probes distance was set to $3.5 \mathrm{~cm}$. In the present study, the measurements were performed using 10 channels positioned over the SM1 and the rostral PFC areas (Fig. 1) according to the modified international EEG 10-10 system (AES, 1994). The probes were mounted on a custom-made cap fixated by several bands surrounding the head of the subject. In order to check the positions of the probes around the motor areas, the subjects were asked to perform a short sequence of rhythmic movements of the contralateral hand to induce a hemodynamic response. If no oxygenation change occurred, the probes were moved several millimeters until, by trial and error, a consistent oxygenation response was obtained. During the placement of the probes, the Oxysoft software (V6.0, Artinis, The Netherlands) allowed real time assessment of the quality of the NIRS signal for each channels based on the light source power level and the receiver gain. In order to demarcate the stimulus periods (i.e., presentation of target force levels), specific events were set indicating the task and rest periods in each block.

\section{Data analysis}

\section{Force levels}

For each subject, the average of the three peak-to-peak amplitudes calculated within a 2-second time window during MVCs was considered as the maximal voluntary force. Target forces in \% MVC were computed subsequently for each subject. After the experimental procedure, each level of desired force was re-calculated over the last

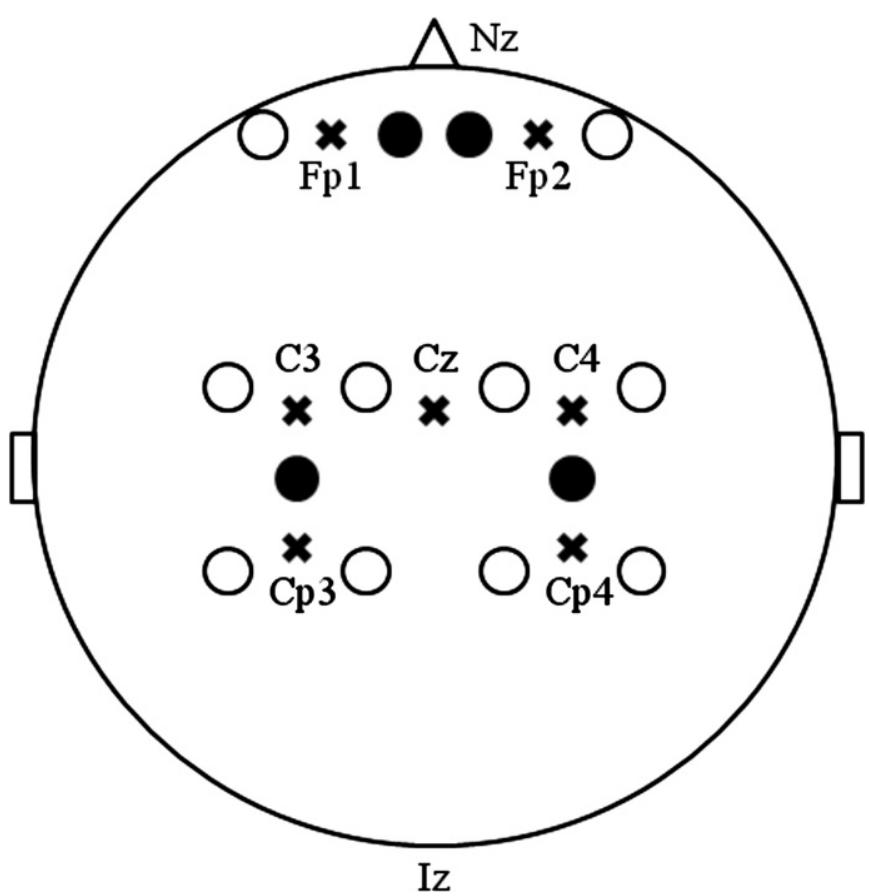

Fig. 1. NIRS probes placement. Each SM1 area was explored by the mean of 4 emitters (empty circles) and one receiver (black circles). The receivers were placed over the contralateral and the ipsilateral SM1 areas according to the modified internationa EEG $10-10$ system between the $\mathrm{C} 3$ and the $\mathrm{Cp} 3$ points, and the $\mathrm{C} 4$ and the $\mathrm{Cp} 4$ points, respectively. Each rostral PFC area was explored by the mean of one emitter and one receiver. The midway between the receivers and the emitters were placed over the Fp1 (contralateral side) and Fp2 (ipsilateral side) points. This placement, resulting in a total of 10 channels, allowed measurement of the activation over the contralateral and ipsilateral SM1 and rostral PFC areas.
$20 \mathrm{~s}$ of force production to check if the subjects followed the task requirement.

\section{Electromyography}

The raw sEMG signals were band-pass filtered (30-500 Hz) before a root mean square (RMS) measure was calculated over the last $20 \mathrm{~s}$ of force production for each target force. This processing was performed using scientific computing software (Scilab 5.3, Consortium Scilab, INRIA-ENPC, Paris, France).

\section{Near infrared spectroscopy}

Dedicated NIRS analysis software was used to obtain $\left[\mathrm{O}_{2} \mathrm{Hb}\right]$ and $[\mathrm{HHb}]$ signals. The raw data were processed offline using the Oxysoft analysis program (V6.0, Artinis, The Netherlands) developed with the multichannel NIRS Oxymon Mk III system. The first step consisted of a visual analysis incorporating pre-processing. This aimed to delete the specific channel where large movement artifacts had occurred or where the signal-to-noise ratio was too low. Then, the NIRS raw data were low-pass filtered using a cut-off frequency of $0.7 \mathrm{~Hz}$ in order to remove the heart rate signal (Huppert et al., 2009). Finally, the data were block averaged and detrended (to eliminate slow drifts) to obtain a grand-average evoked response for each level of force. From the resulting individual signals, the averages of the last $20 \mathrm{~s}$ of each resting period preceding the stimulation period under consideration were subtracted from the averages of the last $5 \mathrm{~s}$ of each stimulation period (Colier et al., 1999). This analysis resulted in deltas, noted $\Delta\left[\mathrm{O}_{2} \mathrm{Hb}\right]$ and $\Delta[\mathrm{HHb}]$. For each level of force, the $\Delta\left[\mathrm{O}_{2} \mathrm{Hb}\right]$ and $\Delta[\mathrm{HHb}]$ for the four channels over the SM1 areas were averaged together resulting in an overall SM1 response. In order to determine left/right asymmetry of rostral PFC and SM1 activity during the handgrip tasks, a laterality index for $\Delta\left[\mathrm{O}_{2} \mathrm{Hb}\right]$ changes was calculated for each level of target force, as follows: (left side - right side)/ (left side + right side) (Cramer et al., 2002; Lee et al., 2000).

\section{Statistical analysis}

Statistica software (version 7.0, Statsoft, Oklahoma, United-States) was used for all analyses. All data were examined for normality and homogeneity using Skewness-Kurtosis and Levene tests, respectively. A two-way repeated measures ANOVA was used to test for any significant effect of the hemisphere side (ipsilateral, contralateral) and the level of force $\left(5,10,20,30,40,50 \%\right.$ of MVC) on the changes in $\Delta\left[\mathrm{O}_{2} \mathrm{Hb}\right]$ and $\Delta[\mathrm{HHb}]$ over the SM1 and the rostral PFC areas. When appropriate, the Tukey's HSD post-hoc test was used to detect paired differences. A student's $t$-test for paired samples was used to test for difference between the predicted and the measured levels of force. Because of the non-normal distribution of the EMG RMS and MVC samples, nonparametric tests have been applied on these data. Friedman ANOVAs were performed to test for the significant effect of the level of force on the laterality index and the EMG RMS values. Similarly, Friedman ANOVA was performed to test for any change in the MVC values between each block. When appropriate, the Conover post-hoc test was used to detect paired differences. The significance level was set at $\mathrm{p}<.05$. Data are presented mean \pm standard deviation (SD) unless specified.

\section{Results}

\section{MVC and target force levels}

A Student's t-test for paired samples ensured that there was no difference between predicted and measured levels of force $(t=0.04$, $\mathrm{p}=.96)$. The MVCs realized after each block were $260.8 \pm 67.1 \mathrm{~N}$, $269.6 \pm 64.8 \mathrm{~N}$ and $262.7 \pm 70.6 \mathrm{~N}$ and were not significantly different from one another $\left(\mathrm{Fr}_{15,2}=1.6, \mathrm{p}=.45\right)$. 


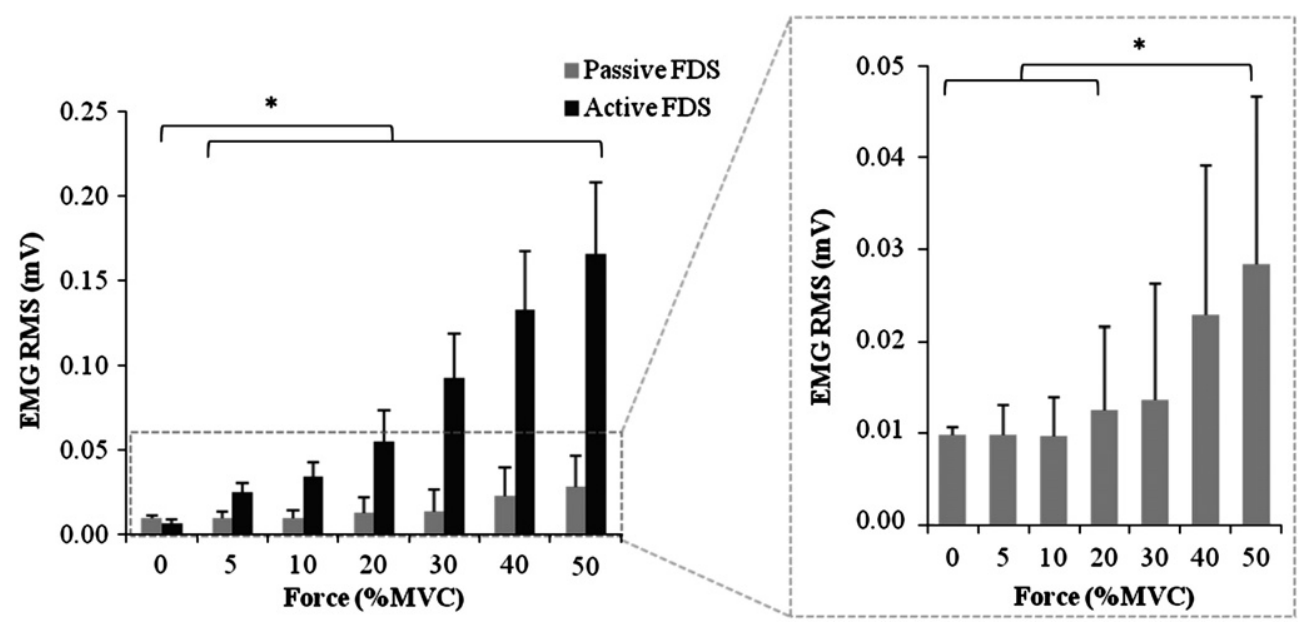

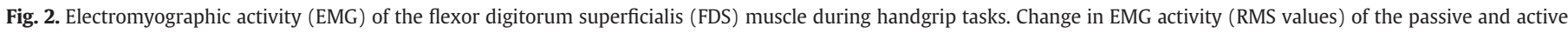

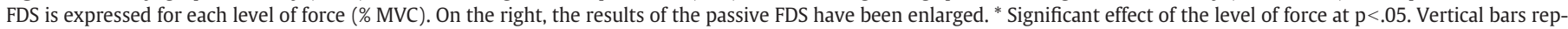
resent SE.

\section{Electromyography}

A significant increase in EMG RMS values was observed as a function of the force levels in both the active $\left(\mathrm{Fr}_{15,6}=84.21 ; \mathrm{p}<.001\right)$ and passive FDS $\left(\mathrm{Fr}_{15,6}=31.25 ; \mathrm{p}<.001\right)$. The Conover post-hoc tests indicated a significant increase in the EMG RMS of the active FDS beyond $10 \%$ MVC $(\mathrm{p}<.05)$ and a significantly higher EMG RMS of the passive FDS at $50 \%$ MVC compared to $0,5,10$ and $20 \%$ of MVC was found ( $<<.05$; Fig. 2).

\section{Cortical activation and force}

All trials were similar in terms of cortical activation response, typically expressed as an increase in $\left[\mathrm{O}_{2} \mathrm{Hb}\right]$ and a slight or no decrease in [HHb] (Hirth et al., 1996; Obrig et al., 2000). However, because of motion artifacts and a low signal-to-noise ratio in some cases, $7.1 \%$ of the SM1 signals and $21.6 \%$ of the rostral PFC signals were removed from the analysis.

\section{Changes in the contralateral and ipsilateral SM1 areas}

A significant increase in $\Delta\left[\mathrm{O}_{2} \mathrm{Hb}\right]$ with the increased levels of force $\left(\mathrm{F}_{5,60}=14.26, \mathrm{p}<.001\right)$ was observed over the SM1 areas but there was no difference according to the hemisphere side $\left(F_{1,12}=3.90\right.$, $\mathrm{p}>.05)$. Post-hoc tests indicated a significant increase in $\Delta\left[\mathrm{O}_{2} \mathrm{Hb}\right]$ between $30,40,50 \%$ of MVC and 5\% of MVC and 40 and 30\% of MVC. No difference in $\triangle\left[\mathrm{O}_{2} \mathrm{Hb}\right]$ was found between 40 and $50 \%$ of MVC on both hemispheres. A significant increase in $\Delta[\mathrm{HHb}]$ with the increased level of force $\left(F_{5,65}=6.35, p<.001\right)$ was also observed with no change with respect to the hemisphere side $\left(\mathrm{F}_{1,13}=3.23, \mathrm{p}=.09\right)$. Post-hoc tests indicated a significant increase in $\Delta[\mathrm{HHb}]$ beyond $40 \%$ of MVC on both hemispheres. These results are presented in Fig. 3.A.

\section{Changes in the contralateral and ipsilateral rostral PFC areas}

A significant interaction of force level $\times$ hemisphere was found in $\Delta\left[\mathrm{O}_{2} \mathrm{Hb}\right]\left(\mathrm{F}_{5,45}=3.29, \mathrm{p}<.05\right)$. Post-hoc tests indicated significant increase in $\Delta\left[\mathrm{O}_{2} \mathrm{Hb}\right]$ between $30,40,50 \%$ of MVC and $5 \%$ of MVC, 40 and $30 \%$ of MVC and 50 and $40 \%$ of MVC in both sides. A significant difference in $\Delta\left[\mathrm{O}_{2} \mathrm{Hb}\right]$ was found between the contralateral and the ipsilateral rostral PFC at 50\% MVC $(\mathrm{p}<.001) . \Delta[\mathrm{HHb}]$ increased significantly with the increased level of force $\left(\mathrm{F}_{5,20}=12.01, \mathrm{p}<.001\right)$ but with no difference between the two hemispheres $\left(\mathrm{F}_{1,4}=0.29, \mathrm{p}=.62\right)$. Post-hoc tests indicated a significant increase in $\triangle[\mathrm{HHb}]$ beyond $40 \%$ of MVC on both hemispheres. These results are presented in the Fig. 3.B.
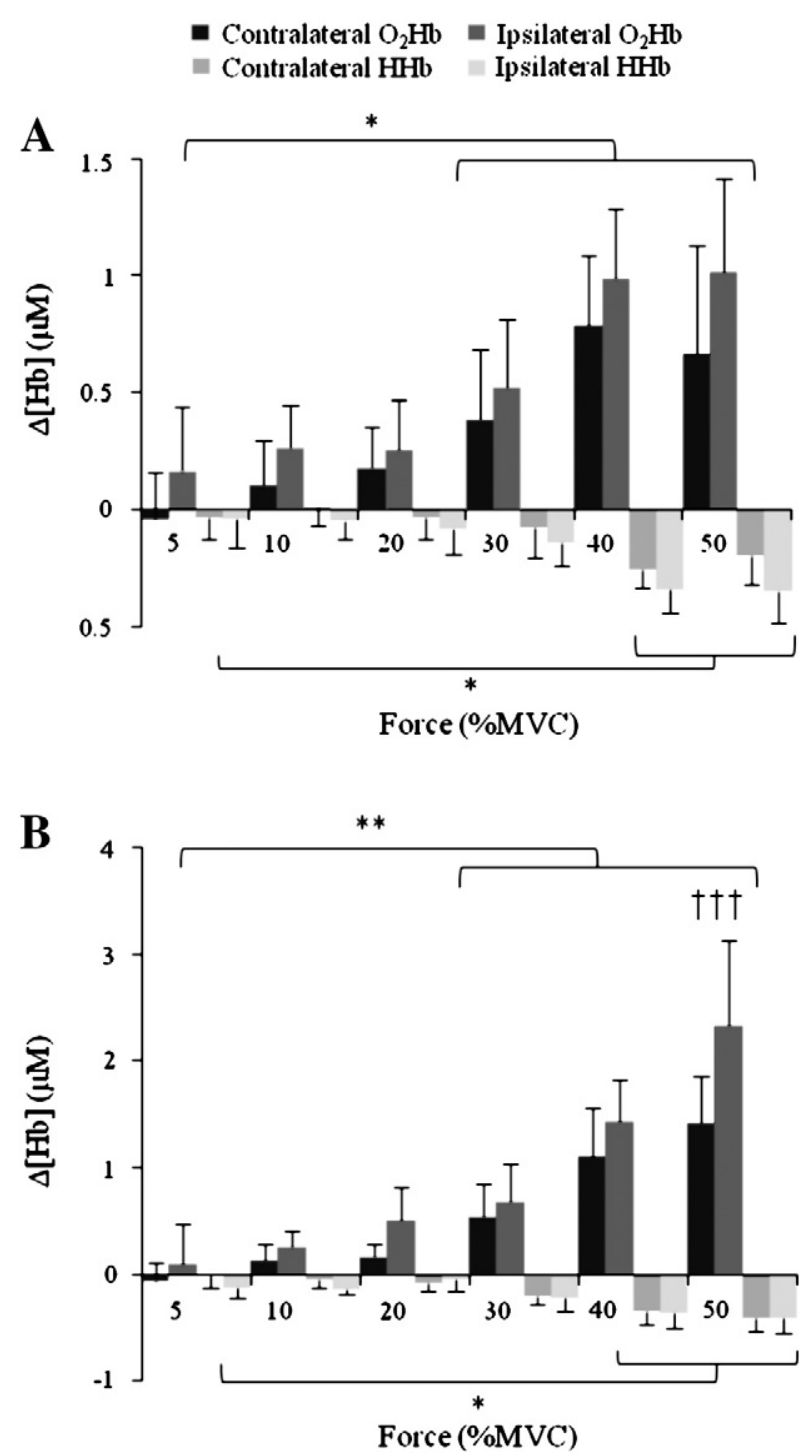

Fig. 3. Cortical activation during handgrip tasks. Changes in the contralateral and ipsilateral SM1 (A) and rostral PFC (B) areas are expressed for each level of force (\% MVC). Significant effect of the level of force ${ }^{*} \mathrm{p}<.05,{ }^{* *} \mathrm{p}<.01$. Significant effect of the hemisphere: $+\dagger \mathrm{p}<.001$. Vertical bars represent SE. 
Laterality indexes

No significant difference was found between the laterality indexes of the SM1 areas for each level of target force $\left(F r_{14,5}=2.27, \mathrm{p}=.81\right)$. Due to the removal of some rostral PFC data, the calculation of the laterality indexes for this area was performed on 9 subjects. No significant difference was found between the laterality indices of the rostral PFC areas for each level of force $\left(\mathrm{Fr}_{9,5}=3.37, \mathrm{p}=.62\right)$.

\section{Discussion}

The aim of this study was to highlight the responses of the ipsilateral and contralateral SM1 and rostral PFC areas for graded levels of force during a unilateral handgrip task. We found that, at increasing levels of handgrip force from 5 to $50 \%$ of MVC, (i) the cortical activation responses over the SM1 areas did not differ between the contralateral and ipsilateral hemispheres while (ii) the rostral PFC activation was significantly higher on the ipsilateral side at the highest force (i.e., 50\% of MVC); (iii) there was a significant increase in the EMG activity of the passive arm when producing 50\% of MVC. These findings are examined in detail next.

\section{Changes in the SM1 areas' activity}

First of all, it is notable that all the investigated areas showed a significant cortical activation in producing static force beyond 30\% of MVC. These results corroborate with earlier studies that investigated the relationship between the levels of force and the cortical activation either using NIRS (Derosiere and Perrey, 2012) or fMRI (Thickbroom et al., 1998) techniques. Further, the lack of significant increase in contralateral cortical activity at low levels of force has been previously described in primates (Cheney and Fetz, 1980; Georgopoulos et al., 1992) and humans (Ludman et al., 1996).

A second observation of note was that SM1 activation reached a plateau at around $40 \%$ of MVC. A similar result was found by Mima et al. (1999) who showed a decrease in the EEG signal power in the beta band between 40 and $60 \%$ of MVC, and between 60 and $80 \%$ of MVC. This prompts the interesting question as to: how is the production of a higher EMG output possible if the contralateral SM1 area activation remains stable or even decreases between 40 and $50 \%$ of MVC? We suggest that the secondary motor areas could participate in producing the increased drive required for higher levels of force. Indeed, according to Dum and Strick (2002), these areas have direct connections with spinal motoneurons, particularly those innervating hand muscles, and may therefore directly generate motor command during a handgrip task.

A third result of interest is that the ipsilateral SM1 area was found to be activated to the same degree as the contralateral SM1 area at all investigated levels of force. This result is further reinforced by the absence of modification of the laterality index at increasing levels of force and strongly supports the findings of Dai et al. (2001). Also, this result is in line with the symmetrical M1 activation found during the course of a low-intensity fatiguing handgrip task in the study of Shibuya et al. (2008). We do stress here a central issue of the present study. Symmetrical cortical activation is not restricted only to complex sequential finger movements but also occurs during static handgrip contractions. Nonetheless, the symmetrical SM1 activation occurrence in response to unilateral handgrip tasks may be subject to certain conditions. Some authors have failed to find symmetrical SM1 activation either during power-handgrip tasks (Cramer et al., 2002) or during simple finger tapping tasks (Durduran et al., 2004; Obrig et al., 1996). Differences between studies likely rely on the nature of the experimental setting associated to the task. In the present study and in the studies of Dai et al. (2001) and Shibuya et al. (2008) the handgrip tasks consisted in tracking a line by focusing on a screen in real time conditions. Such visual feedback was not given in the investigations by Cramer et al. (2002), Obrig et al. (1996) and Durduran et al. (2004). By comparing the fMRI-measured brain activity in subjects performing a visuo-guided handgrip task versus a non-guided handgrip task, Kuhtz-Buschbeck et al. (2008) compellingly evidence emerges that performing a visuo-guided handgrip task involves the activity of many additional areas, such as the ipsilateral SM1. In similar vein, Davare et al. (2007) demonstrated that the disruption of ipsilateral M1 activity by means of transcranial magnetic stimulation during a visuo-guided pinching force production altered dramatically the maintenance of the level of force. As mentioned in the introduction, the authors concluded that the ipsilateral M1 area has a role in "shaping precisely" the muscular command originating from the contralateral hemisphere. We propose that a visuo-guided handgrip task requires such a cross-talk between ipsilateral and contralateral SM1 areas. Further, given the linear relationship between force variability and force production (Missenard et al., 2008), at increasing force levels the force output might require significant control to match with the visual target, probably involving an increasing engagement of the ipsilateral SM1 area to shape the muscular command. Otherwise, as underlined by van Wijk et al. (2012), the ipsilateral M1 activity does not only result from cross-talk between hemispheres aiming at driving the muscular command. Rather, van Wijk et al. (2012) suggested that the ipsilateral and contralateral M1 activity may have distinct roles. We proposed thereafter two potential explanations of the symmetrical activation of the SM1 areas during unimanual handgrip tasks.

First, it can be speculated that the ipsilateral CST acted in the performed task to shape the intended levels of force. In this case, the higher number of excitatory post-synaptic potentials (PSP) onto the pyramidal neurons of the ipsilateral SM1 area would finally generate action potentials throughout the ipsilateral CST. However, the ipsilateral CST constitutes only five to six percent of the total of CST nervous fibers (Brosamle and Schwab, 1997). Therefore, it can be assumed that the number of excitatory PSP within the ipsilateral SM1 area would be reduced compared to those within the contralateral SM1 area and consequently the involvement of the ipsilateral CST cannot alone explain the symmetrical activation of the SM1 areas.

The second explanation concerns the occurrence of interhemispheric inhibition (IHI). The IHI is a mechanism responsible for the inhibition of mirror movements of the passive limb during unilateral hand movements (for a review, see Kicic et al., 2008). It is believed that the IHI consists of the depolarization of trans-colossal glutamatergic nervous fibers originating in the contralateral SM1, which consequently depolarize inhibitory inter-neurons within the ipsilateral SM1 area. These inter-neurons in turn inhibit the pyramidal neurons projecting to the contralateral arm (i.e., the passive arm). It is interesting to note that we observed a tendency of the ipsilateral SM1 area to be more activated than the contralateral SM1 area (Fig. 3), although this effect did not reach statistical significance. Based on this viewpoint, it can be supposed that the summation of the excitatory PSP from the trans-colossal nervous fibers and the inhibitory PSP from the inter-neurons is the cause of the stronger ipsilateral activation. More precisely, the IHI was found higher when performing 1 to $2 \%$ of MVC than when performing 20 to $40 \%$ of MVC during a pinch grip task (Liepert et al., 2001). Therefore, the reasoning provided above as regards to the potential role of the IHI in increasing the ipsilateral SM1 area activation is more valid in the interpretation of the symmetrical cortical activation occurring below 20\% of MVC and not above this level of force. This result is further reinforced by considering the relative stability of the EMG activity of the passive FDS below $20 \%$ of MVC.

\section{Changes in the rostral PFC areas' activity}

We argue in the introduction that the rostral PFC areas should be symmetrically activated with increasing levels of force because they are ipsilaterally connected to the motor areas through cortico-cortical pathways (Krieghoff et al., 2011) and involved in the initiation and 
the control of voluntary movements (Miller and Cohen, 2001). Contrary to the SM1 areas, the difference between the activation of the ipsilateral and the contralateral rostral PFC areas reached statistical significance at $50 \%$ of MVC, in favor of a more intense ipsilateral activation. A hypothesis to explain this result is that the ipsilateral rostral PFC area (the right rostral PFC in the present study) was more activated because of the sustained attention required to perform the task. The right rostral PFC area is well-known to be responsible for the control of sustained attention (Sturm and Willmess, 2001). In similar vein, Okamoto et al. (2004) found a right-sided increase in rostral PFC activation during a complex manual task requiring attentional control (i.e., task consisted of peeling an apple) and suggested an involvement of the executive functions to perform the task. We suggest that the visuo-guided motor task contained in our protocol which required that the subject precisely maintained a target on the screen over a $30 \mathrm{~s}$ period involved a high level of sustained attention.

\section{Changes in EMG activity}

As has been described in the literature (e.g., Woods and Bigland-Ritchie, 1983), the EMG activity of the muscles involved in the task (i.e., the ipsilateral FDS) increases proportionally to the force produced during the isometric contractions. In terms of the EMG activity of the passive FDS measured in this study, a significant increase was found at $50 \%$ of MVC (Fig. 2). A similar result was found by Van Duinen et al. (2008) at 70\% of MVC and can be linked to the study of Liepert et al. (2001) cited above who showed a lower IHI when increasing the level of force. These authors interpreted the occurrence of stronger IHI during unilateral hand movements at low levels of force as linked to the need to improve the dexterity during fine finger movements. They hypothesized that the inhibition of ipsilateral pyramidal neurons would therefore reduce the risk of interference of both hands, improving therefore the precision during handling of objects. According to this hypothesis about the role of the IHI during the production of higher levels of force, we suggest that the nervous system would adapt itself and would consequently not inhibit the passive arm explaining the occurrence of "mirror" contractions of the FDS at 50\% of MVC.

\section{Study limitations}

Although the present study revealed interesting results on the bilateral activation patterns related to the control of submaximal force generation, it was nevertheless subject to a number of limitations. First, a potential limitation may be related to the anatomical positioning of the optodes. As most studies did, we used the international 10-10 system for EEG recordings (and skull surface landmarks) to localize the target cortex and position the NIRS optodes. Subsequently, functional oxygenation was controlled by executing a simple task to reveal hemodynamic responses. If no hemodynamic change was detected, the optodes were moved several millimeters and the task was executed again. In the present study, this was repeated until a consistent hemodynamic response was found (Shibuya et al., 2008). Also, we focused on decreases in [HHb] to ensure the correct location of the channel (Kleinschmidt et al., 1996). The location of NIRS recordings can therefore generally only be assumed to have correctly covered the targeted areas. Second, we have to consider some important potential drawbacks regarding the $\left[\mathrm{O}_{2} \mathrm{Hb}\right]$ signals. Indeed, recent studies (e.g., Kirilina et al., 2012; Takahashi et al., 2011) have raised the question of the superficial (i.e., extra-cortical) contributions to NIRS signals measured at the scalp level. Among them, Kirilina et al. (2012) have notably evidenced that the superficial contributions are more pronounced in the $\left[\mathrm{O}_{2} \mathrm{Hb}\right]$ and total hemoglobin signals, but not in the $[\mathrm{HHb}]$. The authors suggested that looking at the changes [HHb] may allow identification of false positive in NIRS activation maps (i.e., erroneously attributing NIRS responses to cortical changes). In the present study, we found changes in both $\left[\mathrm{O}_{2} \mathrm{Hb}\right]$ and $[\mathrm{HHb}]$ which brings some confidence as regards the attribution of the observed NIRS responses to cortical changes. Note that the $\Delta[\mathrm{HHb}]$ changed significantly only above $40 \%$ of MVC for each cortical area investigated in this study. This can be explained by the existence of smaller changes in $[\mathrm{HHb}]$ compared to that in $\left[\mathrm{O}_{2} \mathrm{Hb}\right]$ during neurovascular coupling - a phenomenon well "represented" by the balloon model (Buxton and Frank, 1997). Future investigations using methods to definitively separate cortical and extracortical signals in NIRS signals are warranted. These methods notably include the use of additional short source-detector separation optodes as regressors (Gagnon et al., 2012; Saager et al., 2011) and the analysis of the photon time-of-flight distribution in time-domain NIRS (Aletti et al., 2012).

\section{Conclusion}

In summary, our results indicate a bilateral activation over the SM1 and rostral PFC areas during a unilateral handgrip task for graded levels of force. In addition to this, the symmetry of the activation was demonstrated in all investigated levels of force concerning the SM1 areas, showing therefore that such a pattern is not limited to complex finger movements. We suggest that the visuo-guided control of force levels during a handgrip task requires cross-talk from ipsi- to contralateral SM1 to cope for the relative complexity of the task, similarly to that observed during complex sequential finger movement. We also proposed alternative ways to explain the symmetrical SM1 activation including (i) the ipsilateral CST and (ii) IHI mechanism. In addition, the more intense ipsilateral (right) rostral PFC activation compared to the contralateral (left) at the highest level of force reflects the higher attentional demand required to control the motor output. To better understand the symmetrical, or at least bilateral, cortical activation during unilateral handgrip tasks, the possibility of measuring inhibitory and excitatory inputs must be strongly considered.

\section{Acknowledgment}

The authors would like to thank the Languedoc-Roussillon Region council (AVENIR) for funding the NIRS equipment and the French University Institute (IUF) in supporting this work. Further, we would like to thank Dr. Jean-Paul MICALLEF for his assistance in the development of experimental materials.

\section{References}

Aletti, F, Re, R, Pace, V, Contini, D., Molteni, E, Cerutti, S, Maria Bianchi, A, Torricelli, A., Spinelli, L., Cubeddu, R., Baselli, G., 2012. Deep and surface hemodynamic signal from functional time resolved transcranial near infrared spectroscopy compared to skin flow motion. Comput. Biol. Med. 42, 282-289.

American Electroencephalographic Society, 1994. Guideline thirteen: guidelines for standard electrode position nomenclature. J. Clin. Neurophysiol. 11, 111-113.

Bawa, P., Hamm, J.D., Dhillon, P., Gross, P.A., 2004. Bilateral responses of upper limb muscles to transcranial magnetic stimulation in human subjects. Exp. Brain Res. 158 385-390.

Brosamle, C., Schwab, M.E., 1997. Cells of origin, course, and termination patterns of the ventral, uncrossed component of the mature rat corticospinal tract. J. Comp. Neurol. 386, 293-303.

Brus-Ramer, M., Carmel, J.B., Martin, J.H., 2009. Motor cortex bilateral motor representation depends on subcortical and interhemispheric interactions. J. Neurosci. 29 6196-6206.

Buxton, R.B., Frank, L.R., 1997. A model for the coupling between cerebral blood flow and oxygen metabolism during neural stimulation. J. Cereb. Blood Flow Metab. 17, 64-72.

Catalan, M.J., Honda, M., Weeks, R.A., Cohen, L.G., Hallett, M., 1998. The functional neuroanatomy of simple and complex sequential finger movements: a PET study. Brain 121, 253-264

Cheney, P.D., Fetz, E.E., 1980. Functional classes of primate corticomotoneuronal cells and their relation to active force. J. Neurophysiol. 44, 773-791.

Colier, W.N.J.M., Quaresima, V., Oeseburg, B., Ferrari, M., 1999. Human motor-cortex oxygenation changes induced by cyclic coupled movements of hand and foot Exp. Brain Res. 129, 457-461.

Cramer, S.C., Weisskoff, R.M., Schaechter, J.D., Nelles, G., Foley, M., Finklestein, S.P., Rosen, B.R., 2002. Motor cortex activation is related to force of squeezing. Hum. Brain Mapp. 16, 197-205. 
Dai, T.H., Liu, J.Z., Sahgal, V., Brown, R.W., Yue, G.H., 2001. Relationship between muscle output and functional MRI-measured brain activation. Exp. Brain Res. 140, 290-300.

Davare, M., Duque, J., Vandermeeren, Y., Thonnard, J.L., Olivier, E., 2007. Role of the ipsilateral primary motor cortex in controlling the timing of hand muscle recruitment. Cereb. Cortex 17, 353-362.

Delpy, D.T., Cope, M., Van Der Zee, P., Arridge, S., Wrayt, S., Wyatt, J.D., 1988. Estimation of optical pathlength through tissue from direct time of flight measurement. Phys. Med. Biol. 33, 1433-1442.

Derosiere, G., Perrey, S., 2012. Relationship between submaximal handgrip muscle force and NIRS-measured motor cortical activation. Adv. Exp. Med. Biol. 737, 269-274.

Dum, R.P., Strick, P.L., 2002. Motor areas in the frontal lobe of the primate. Physiol. Behav. 77, 677-682.

Duncan, L.A., Wildsmith, J.A., Ruckley, C.V., 1996. Near infrared spectroscopy. Anaesthesia $51,710-711$.

Durduran, T., Yu, G., Burnett, M.G., Detre, J.A., Greenberg, J.H., Wang, J., Zhou, C., Yodh, A.G., 2004. Diffuse optical measurement of blood flow, blood oxygenation, and metabolism in a human brain during sensorimotor cortex activation. Opt. Lett. 29 1766-1768.

Ehrsson, H.H., Fagergren, A., Jonsson, T., Westling, G., Johansson, R.S., Forssberg, H., 2000. Cortical activity in precision- versus power-grip tasks: an fMRI study. J Neurophysiol. 83, 528-536.

Elwell, C.E., Cope, M., Edwards, A.D., Wyatt, J.S., Delpy, D.T., Reynolds, E.O., 1994. Quantification of adult cerebral hemodynamics by near-infrared spectroscopy. J. Appl. Physiol. 77, 2753-2760.

Gagnon, L., Cooper, R.J., Yücel, M.A., Perdue, K.L., Greve, D.N., Boas, D.A., 2012. Short separation channel location impacts the performance of short channel regression in NIRS. Neuroimage 59, 2518-2528.

Georgopoulos, A.P., Ashe, J., Smyrnis, N., Taira, M., 1992. The motor cortex and the coding of force. Science 256, 1692-1695.

Hirth, C., Obrig, H., Villringer, K., Thiel, A., Bernarding, J., Muhlnickel, W., Flor, H. Dirnagl, U., Villringer, A., 1996. Non-invasive functional mapping of the human motor cortex using near-infrared spectroscopy. Neuroreport 7, 1977-1981.

Huppert, T.J., Diamond, S.G., Franceschini, M.A., Boas, D.A., 2009. HomER: a review of time-series analysis methods for near-infrared spectroscopy of the brain. Appl. Opt. 48, D280-D298.

Kawashima, R., Itoh, H., Ono, S., Satoh, K., Furumoto, S., Gotoh, R., Koyama, M. Yoshioka, S., Takahashi, T., Takahashi, K., Yanagisawa, T., Fukuda, H., 1996. Changes in regional cerebral blood flow during self-paced arm and finger movements. A PET study. Brain Res. 716, 141-148.

Kicic, D., Lioumis, P., Ilmoniemi, R.J., Nikulin, V.V., 2008. Bilateral changes in excitability of sensorimotor cortices during unilateral movement: combined electroencephalographic and transcranial magnetic stimulation study. Neuroscience 152, 1119-1129.

Kirilina, E., Jelzow, A., Heine, A., Niessing, M., Wabnitz, H., Brühl, R., Ittermann, B. Jacobs, A.M., Tachtsidis, I., 2012. The physiological origin of task-evoked systemic artefacts in functional near infrared spectroscopy. Neuroimage 61, 70-81.

Kleinschmidt, A., Obrig, H., Requardt, M., Merboldt, K.D., Dirnagl, U., Villringer, A Frahm, J., 1996. Simultaneous recording of cerebral blood oxygenation changes during human brain activation by magnetic resonance imaging and near-infrared spectroscopy. J. Cereb. Blood Flow Metab. 16, 817-826.

Krieghoff, V., Waszak, F., Prinz, W., Brass, M., 2011. Neural and behavioral correlates of intentional actions. Neuropsychologia 49, 767-776.

Kuhtz-Buschbeck, J.P., Gilster, R., Wolff, S., Ulmer, S., Siebner, H., Jansen, O., 2008. Brain activity is similar during precision and power gripping with light force: an fMR study. Neuroimage 40, 1469-1481.

Kuypers, H.G.J.M., 1985. The anatomical and functional organization of the motor system. In: Swash, M., Kennard, C. (Eds.), Scientific Basis of Clinical Neurology. Churchill Livingstone, London, pp. 3-18.

Lee, M., Reddy, H., Johansen-Berg, H., Pendlebury, S., Jenkinson, M., Smith, S., Palace, J. Matthews, P.M., 2000. The motor cortex shows adaptive functional changes to brain injury from multiple sclerosis. Ann. Neurol. 47, 606-613.

Liepert, J., Dettmers, C., Terborg, C., Weiller, C., 2001. Inhibition of ipsilateral motor cortex during phasic generation of low force. Clin. Neurophysiol. 112, 114-121.

Liu, J.Z., Shan, Z.Y., Zhang, L.D., Sahgal, V., Brown, R.W., Yue, G.H., 2003. Human brain activation during sustained and intermittent submaximal fatigue muscle contractions: an FMRI study. J. Neurophysiol. 90, 300-312.

Ludman, C.N., Cooper, T.G., PloutzSynder, L.L., Potchen, E.J., Meyer, R.A., 1996. Force of voluntary exercise does not affect sensorimotor cortex activation as detected by functional MRI at 1.5 T. NMR Biomed. 9, 228-232.
Miller, E.K., Cohen, J.D., 2001. An integrative theory of prefrontal cortex function. Annu. Rev. Neurosci. 24, 167-202.

Mima, T., Simpkins, N., Oluwatimilehin, T., Hallett, M., 1999. Force level modulates human cortical oscillatory activities. Neurosci. Lett. 275, 77-80.

Missenard, O., Mottet, D., Perrey, S., 2008. The role of cocontraction in the impairment of movement accuracy with fatigue. Exp. Brain Res. 185, 151-156.

Mottola, L., Crisostomi, S., Ferrari, M., Quaresima, V., 2006. Relationship between handgrip sustained submaximal exercise and prefrontal cortex oxygenation. Adv. Exp. Med. Biol. 578, 305-309.

Napier, J.R., 1956. The prehensile movements of the human hand. J. Bone Joint Surg. Br. $38,902-913$.

Obrig, H., Hirth, C., Junge-Hulsing, J.G., Doge, C., Wolf, T., Dirnagl, U., Villringer, A., 1996. Cerebral oxygenation changes in response to motor stimulation. J. Appl. Physiol. $81,1174-1183$

Obrig, H., Neufang, M., Wenzel, R.D., Kohl, M., Steinbrink, J., Einhaüpl, K., Villringer, A., 2000. Spontaneous low frequency oscillations of cerebral hemodynamics and metabolism in human adults. NeuroImage 12, 623-639.

Okamoto, M., Dan, H., Shimizu, K., Takeo, K., Amita, T., Oda, I., Konishi, I., Sakamoto, K., Isobe, S., Suzuki, T., Kohyama, K., Dan, I., 2004. Multimodal assessment of cortical activation during apple peeling by NIRS and fMRI. Neuroimage 21, 1275-1288.

Oldfield, R.C. 1971. The assessment and analysis of handedness: the Edinburgh inventory. Neuropsychologia 9, 97-113.

Perrey, S., 2008. Non-invasive NIR spectroscopy of human brain function during exercise. Methods 45, 289-299.

Pfurtscheller, G., Neuper, C., Krausz, G., 2000. Functional dissociation of lower and upper frequency mu rhythms in relation to voluntary limb movement. Clin. Neurophysiol. 111, 1873-1879.

Saager, R.B., Telleri, N.L., Berger, A.J., 2011. Two-detector corrected near infrared spectroscopy (C-NIRS) detects hemodynamic activation responses more robustly than single detector NIRS. Neuroimage 55, 1679-1685.

Shibuya, K., Sadamoto, T., Sato, K., Moriyama, M., Iwadate, M., 2008. Quantification of delayed oxygenation in ipsilateral primary motor cortex compared with contralateral side during a unimanual dominant-hand motor task using near-infrared spectroscopy. Brain Res. 1210, 142-147.

Sturm, W., Willmes, K., 2001. On the functional neuroanatomy of intrinsic and phasic alertness. Neuroimage 14, 76-84.

Takahashi, T., Takikawa, Y., Kawagoe, R., Shibuya, S., Iwano, T., Kitazawa, S., 2011. Influence of skin blood flow on near-infrared spectroscopy signals measured on the forehead during a verbal fluency task. Neuroimage 57, 991-1002.

Tanji, J., Okano, K., Sato, K.C., 1988. Neuronal-activity in cortical motor areas related to ipsilateral, contralateral, and bilateral digit movements of the monkey. J. Neurophysiol. 60, 325-343.

Thickbroom, G.W., Phillips, B.A., Morris, I., Byrnes, M.L., Mastaglia, F.L., 1998. Isometric force-related activity in sensorimotor cortex measured with functional MRI. Exp. Brain Res. 121, 59-64.

Van Duinen, H., Renken, R., Maurits, N.M., Zijdewind, I., 2008. Relation between muscle and brain activity during isometric contractions of the first dorsal interosseus muscle. Hum. Brain Mapp. 29, 281-299.

Van Wijk, B.C.M., Beek, P.J., Daffertshofer, A., 2012. Differential modulations of ipsilateral and contralateral beta (de)synchronization during unimanual force production. Eur. J. Neurosci. 36, 2088-2097.

Verstynen, T., Diedrichsen, J., Albert, N., Aparicio, P., Ivry, R.B., 2005. Ipsilateral motor cortex activity during unimanual hand movements relates to task complexity. J. Neurophysiol. 93, 1209-1222.

Wassermann, E.M., Fuhr, P., Cohen, L.G., Hallett, M., 1991. Effects of transcranial magnetic stimulation on ipsilateral muscles. Neurology 41, 1795-1799.

Wassermann, E.M., Pascualleone, A., Hallett, M., 1994. Cortical motor representation of the ipsilateral hand and arm. Exp. Brain Res. 100, 121-132.

Woods, J.J., Bigland-Ritchie, B., 1983. Linear and non-linear surface EMG/force relationships in human muscles. An anatomical/functional argument for the existence of both. Am. J. Phys. Med. 62, 287-299.

Wriessnegger, S.C., Kurzmann, J., Neuper, C., 2008. Spatio-temporal differences in brain oxygenation between movement execution and imagery: a multichannel nearinfrared spectroscopy study. Int. J. Psychophysiol. 67, 54-63.

Ziemann, U., Ishii, K., Borgheresi, A., Yaseen, Z., Battaglia, F., Hallett, M., Cincotta, M., Wasserman, E.M., 1999. Dissociation of the pathways mediating ipsilateral and contralateral motor-evoked potentials in human hand and arm muscles. J. Physiol. 518, 895-906. 